\title{
Dynamic Solution Code for Structural Analysis upon Joint Element
}

\author{
S.A. Sadrnejad and M. Labibzadeh \\ Department of Civil Engineering, Khaajeh Nassiroddin Toosi University, Tehran, Iran
}

\begin{abstract}
The analysis of large structures mostly requires a complete three dimensional finite element discretization. Stability of such structures includes not only the stability of structure itself but also that of foundation as well as interconnection elements. The general instability of a structure may be affected by two aspects, firstly, an inappropriate preliminary classical design of the structure and inadequate control over the geometry and quality of the materials used for construction. Secondary, the foundation rock and abutments may contain a number of faults and joints which may be unfavorable to stability of the structure. Therefore it is necessary to consider appropriately the influence of interaction between the structure, foundation and abutments under static and dynamic loads. from construction point of view some of the structures such arch dams are divided into vertical blocks, which are separated by vertical joints which are grouted at the later stage. Since the behavior of such and many other structures whose have contraction joints under static and dynamic loads depends largely on those joints, therefore, the presence of structural codes that can recommend the appropriate specifications on modeling these joints, seems to be necessary. The present study contribution deals with the development of three dimensional finite element software including interfacial behavior of joints between different substructures and interaction behavior of structure and foundation to evaluate the safety of the structure subjected to static and dynamic loads. The developed software is verified upon different points of view and the results are in agreement with experiments.
\end{abstract}

Key words: Finite, infinite, joint, element, software, model

\section{INTRODUCTION}

As it is mentioned briefly in the above section this study deals with the development of three dimensional multi-form elements software which from its specific capabilities is that both interfacial behavior between substructures and interaction behavior between structure and its foundation can be appropriately modeled.

For modeling the first behavior a specially developed generalized joint element adopted in the software that its formulation and specifications have been presented in the later section. The last behavior on the other hand is modeled with considering an elastostatic infinite element that its formulation and characteristics can be seen in the next section.

In this article constitutive formulation of the interfacial and infinite elements will be presented and subsequently constructive relationships of the finite element method for analyzing static and dynamic problems are explained.

In the forth section the Newmark's algorithm which is applied in the software for solution of dynamic equilibrium will be prescribed.

The briefly software development is mentioned in the seventh section and finally the verification of the software and conclusions are presented.
Proposed discretization: The following elements are used for the discretization purpose:

a. The body of the 3D.structure was modeled by using solid isoparametric brick Elements ${ }^{[1]}$.

b. The rock footing system and the abutment have been modeled using Coupled finite and elasto static infinite elements ${ }^{[2]}$.

c. The interfacial behavior between vertical blocks represented by specially Developed generalized joint element.

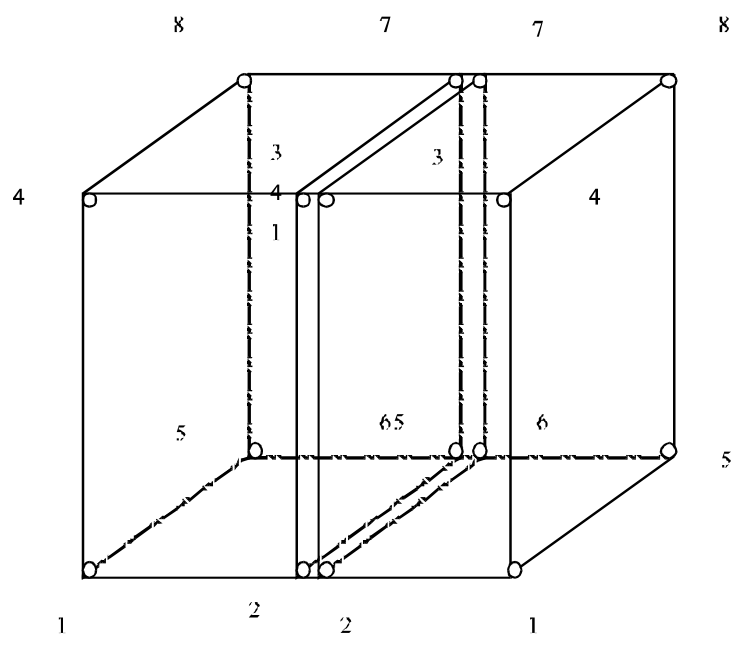

Fig. 1: Shape of joint element between vertical blocks

Corresponding Author: S.A. Sadrnejad, Department of Civil Engineering, Khaajeh Nassiroddin Toosi University, Tehran, Iran 
Formulation of joint element: Since the present element is supposed to be bilinear similar to plane strain and of isoparametric type, Hence the shape functions are written as:

$$
X=\sum_{i=1}^{n} N_{i} X_{i} \quad Y=\sum_{i=1}^{n} N_{i} Y_{i} \quad Z=\sum_{i=1}^{n} N_{i} Z_{i}
$$

$\mathrm{i}=\mathrm{I}$, II, III, IV (No. of nodes per element) and $\mathrm{N}_{\mathrm{i}}$ are the shape functions.

$$
\{\Delta\}=[I,-I]\{\delta\}
$$

$\{\Delta\}$ Is the vector of relative shears and normal displacements in the element, $\mathrm{I}$ is unit square matrix of size $(12 \times 12)$ and finally $\{\delta\}$ is vector of nodal displacements. Upon assuming three degree of displacement at each node, the equation (2) can also be written in the following form:

$$
\{\Delta\}_{12 * 1}=[T]_{12 * 24}\{\delta\}_{24 * 1}
$$

In which $[T]$ is the translation matrices which can be defined from equation (2) and directly depends on the form of employed shape functions.

Here in the case of the interface element with zero thickness, strains can be expressed as follows:

$$
\{\mathcal{E}\}^{T}=\{\Delta u, \Delta v, \Delta w\}^{T}
$$

Note: since the element is mathematically zero thickness hence the strains can be defined as relative displacements. Nevertheless with respect to definition of isoparametric elements we have:

$$
\left\{\begin{array}{l}
\Delta u=\sum_{i=1}^{n} N_{i} \Delta u_{i} \\
\Delta v=\sum_{i=1}^{n} N_{i} \Delta v_{i} \\
\Delta w=\sum_{i=1}^{n} N_{i} \Delta w_{i}
\end{array}\right.
$$

Or in the form of matrix formulation:

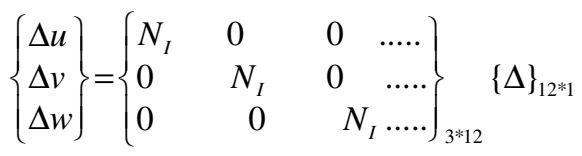

I.E,

$$
\{\varepsilon\}_{3^{* 1}}=[N]_{3^{*} 12}\{\Delta\}_{12^{*} 1}
$$

Using equation (3):

$$
\{\varepsilon\}_{3^{* 1}}=[N]_{3^{* 12}}[T]_{12^{*} 24}\{\delta\}_{24^{* 1}}
$$$$
=\left[B_{J}\right]_{3^{*} 24}\{\delta\}_{24^{* 1}}
$$

Where $\left[\mathrm{B}_{\mathrm{J}}\right]=$ Shape Function matrix for joint element. The general stress -strain relation is expressed as:

$$
\{\sigma\}=[D]\{\varepsilon\} \text { where : }
$$$$
[D]=\left\{\begin{array}{llc}
K_{n n} & 0 & 0 \\
0 & K_{s s} & 0 \\
0 & 0 & K_{s s}
\end{array}\right\}
$$

$\mathrm{K}_{\mathrm{nn}}$ and $\mathrm{K}_{\mathrm{ss}}$ are the normal and shear stiffness respectively.
Finally the stiffness matrix for joint element is defined as follows:

$[K]=\int_{v}\left[B_{J}\right]^{T}[D]\left[B_{J}\right] d v$

Formulation of elasto-static infinite element: Table 1 shows the shape function of an eight node infinite element which is mapped into finite element i.e., $1<\xi<+1$ by using expression upon two known decay forms of $\frac{1}{r}$ and $\frac{1}{\sqrt{r}}$.

$\mathrm{X}=\sum_{i=1}^{n} N_{i} x_{i}$

Table 1: Mapping / shape functions for 3-D isoparametric infinite elements

\begin{tabular}{lc}
\hline \multicolumn{1}{c}{ Mapping/shape Function } \\
\hline$\left(\frac{1}{r}\right)$ type decay & $\left(\frac{1}{\sqrt{r}}\right)$ type decay \\
$\mathrm{Ni}=\frac{\xi \xi_{i}\left(1+\eta \eta_{i}\right)\left(1+\zeta \zeta_{i}\right)}{2(1-\xi)}$ & $\mathrm{Ni}=\frac{1}{3}\left[1-\frac{1}{1-\xi^{2}}\right]\left(1+\eta \eta_{I}\right)\left(1+\zeta \zeta_{I}\right)$ \\
$\mathrm{i}=(\mathrm{I}, \mathrm{II}, \mathrm{III}, \mathrm{IV})$ & $\mathrm{i}=(\mathrm{I}, \mathrm{II}, \mathrm{III}, \mathrm{IV})$ \\
$\mathrm{Ni}=\frac{(1+\xi)\left(1+\eta \eta_{i}\right)\left(1++_{i}\right)}{4(1-\xi)}$ & $\mathrm{Ni}=\frac{1}{12}\left[\frac{4}{(1-\xi)^{2}}-1\right]\left(1+\eta \eta_{i}\right)\left(1+\zeta \zeta_{i}\right)$ \\
$\mathrm{i}=\mathrm{V}, \mathrm{VI}, \mathrm{VII}, \mathrm{VIII}$ & $\mathrm{i}=\mathrm{V}, \mathrm{VI}, \mathrm{VII}, \mathrm{VIII}$
\end{tabular}

In general, the shape function, Ni should satisfy the following conditions:

a. It should have the value of unity at node $i$ and zero at all the other nodes.

b. $\sum_{i=1}^{n} N i=1$

c. For $\xi=+1$ the value of $\mathrm{Ni}$ should tend to infinity and

d. $\quad \sum_{i=1}^{n} \frac{\partial N_{i}}{\partial \xi}=\sum_{i=1}^{n} \frac{\partial N_{i}}{\partial \eta}=\sum_{i=1}^{n} \frac{\partial N_{i}}{\partial \zeta}=o$

Where $\mathrm{n}=$ Number of nodes per element.

The inverse mapping of this element can be expressed using Equation (11) as:

$$
\begin{aligned}
& x=\frac{-\xi(1-\eta)(1+\zeta)}{2(1-\xi)} x_{1} \\
& +\frac{-\xi(1-\eta)(1-\zeta)}{2(1-\xi)} x_{2} \\
& +\frac{-\xi(1+\eta)(1-\zeta)}{2(1-\xi)} x_{3} \\
& +\frac{-\xi(1+\eta)(1+\zeta)}{2(1-\xi)} x_{4} \\
& +\frac{(1+\xi)(1-\eta)(1+\zeta)}{4(1-\xi)} x_{5} \\
& +\frac{(1+\xi)(1-\eta)(1-\zeta)}{4(1-\xi)} x_{6} \\
& +\frac{(1+\xi)(1+\eta)(1-\zeta)}{4(1-\xi)} x_{7} \\
& +\frac{(1+\xi)(1+\eta)(1+\zeta)}{4(1-\xi)} x_{8}
\end{aligned}
$$


Where, $x^{1}$ to $x^{8}$ are the coordinates of nodes 1 to 8 , respectively. Now with

$X^{5}=2 x^{1} ; x^{6}=2 x^{2} ; x^{7}=2 x^{3}$ and $x^{8}=2 x^{4}$

The inverse mapping can be written as follows

$$
\xi=1-\frac{1}{2 x}\left[\begin{array}{l}
\left(x_{1}+x_{2}+x_{3}+x_{4}\right) \\
+\xi\left(x_{1}-x_{2}-x_{3}+x_{4}\right) \\
+\eta\left(-x_{1}-x_{2}+x_{3}+x_{4}\right) \\
+\eta \xi\left(-x_{1}+x_{2}-x_{3}+x_{4}\right)
\end{array}\right]
$$

The condition given by Equation (15) indicates that the middle nodes, $5,6,7$ and 8 should placed at a distance of twice the distance of nodes 1,2,3,4, respectively, from the reference pole. The values of $\xi$ for all the nodes obtained via equation number (16) are tabulated in Table 2. Identical expressions can be worked out for $\eta$ and $\zeta$. The same approach is followed for any other elements with $\frac{1}{r}$ and $\frac{1}{\sqrt{r}}$ types of decay.

\begin{tabular}{llllc} 
Table 2: & \multicolumn{5}{l}{ Values of $\xi$ at various nodes } \\
\hline $\begin{array}{l}\text { Node number } \\
(1)\end{array}$ & $\begin{array}{c}\mathrm{x}_{2} \\
(2)\end{array}$ & $(3)$ & $\begin{array}{l}\zeta \\
(4)\end{array}$ & $\begin{array}{c}\xi \text { obtained from } \\
\text { equation } 16(5)\end{array}$ \\
\hline 1 & $\mathrm{x}_{1}$ & -1 & +1 & -1 \\
2 & $\mathrm{x}_{2}$ & -1 & -1 & -1 \\
3 & & & & \\
4 & $\mathrm{x}_{3}$ & +1 & -1 & -1 \\
5 & $\mathrm{x}_{4}$ & +1 & +1 & -1 \\
6 & $\mathrm{x}_{5}=2 \mathrm{x}_{1}$ & -1 & +1 & 0 \\
7 & $\mathrm{x}_{6}=2 \mathrm{x}_{2}$ & -1 & -1 & 0 \\
8 & $\mathrm{x}_{7}=2 \mathrm{x}_{3}$ & +1 & -1 & 0 \\
\hline
\end{tabular}

Finite element analysis and solution of dynamic equations: Since the inception of finite element method in the late fifties, finite element method has been extensively used by structural analysts to tackle a wide range of continuum mechanics problems. The vigorous research activities sustain in this field and the rapid developments of computer hardware have been extended the structural engineering applications of the method to an unpredicted scale.

Complex structures such as arch dams, nuclear power plants, hyperbolic cooling towers etc. can now be numerically analyzed.

Static or quasi static analysis generally neglects the inertial and damping effects while it is largely under the effect of interfacial locations. However when a structure is subjected to time varying loading such as impact, explosive or seismic loading, these effects are certainly important to a truly dynamic analysis that must perform.

In the following section, the finite element formulation, numerical computations of the mass and appropriate damping matrices is discussed .The principal of virtual work establishes that for a body which is in static/dynamic equilibrium, the variation of the summation of internal and external virtual work with respect to kinematical admissible displacement must be equal to zero ${ }^{[1]}$.
This principal reveals that it can be written as follows:

$\delta W^{\text {int }}-\delta W^{\text {ext }}=0.0$

The internal work is:

$\delta W^{\mathrm{int}}=\int_{v} \delta \varepsilon_{i j} \sigma_{i j} d v$

and the external virtual work is given by:

$\delta W^{e x t}=\int_{A} \delta u_{k} f_{s k} d A+\int_{v} \delta u_{k} z_{k} d v$

In which,

$f_{s k}=$ Surface (Traction) force components.

$z_{k}=$ Equivalent volume force components.

$\delta u_{k}=$ Virtual displacements.

$\delta \varepsilon_{i j}=$ Virtual strains associated with $\delta u_{k}$

Now, decomposing $z_{k}$ into its constituent parts, it is possible to write:

$z_{k}=f_{b k}-\rho \ddot{u}_{k}-c \dot{u}_{k}$

Where, $f_{b k}, \rho \ddot{u}_{k}$ and $c \dot{u}_{k}$ are respectively, the body,

inertial and damping forces and $\rho$ is the unit mass; c is the damping parameters and dots denoting the differentiating with respect to time.

The variation of energy equation may finally be written as follows:

$\int_{v} \delta \varepsilon_{i j} \sigma i j d v-\int_{v} \delta u_{k}\left(f_{b k}-\rho \ddot{\mathrm{u}}_{\mathrm{k}}-c \dot{u}_{k}\right) d v$

$-\int_{A} \delta u_{k} f_{s k} d_{A}=0.0$

From the finite element point of view the above equation can be re-written as:

$\int_{v} \delta \varepsilon_{i j} \sigma i j d v=\{d \delta\}^{T} \int_{v}[B]^{T}[D][B] d v\{\delta\}$

$\int_{v} \delta u_{k}\left(f_{b k}-f \dot{\mathrm{u}}_{\mathrm{k}}-c u_{k}\right) d v$

$=\{d \delta\}^{T}\left(\int_{v}[N]^{T}\left\{f_{b}\right\} d v-\int_{v}[N]^{T} \rho[N]\{\ddot{\mathrm{u}}\} d v\right.$

$\left.-\int_{v}[N]^{T}[c][N]\{\dot{u}\} d v\right)$

$\int_{A} \delta u_{k} f_{s k} d A=\{d \delta\}^{T} \int_{A}[N]^{T}\left\{f_{s}\right\} d A$

This equation in matrix form is the known dynamic equilibrium as follows:

$[M]\{\ddot{u}\}+[C]\{\dot{u}\}+[K]\{u\}=\left\{f_{(t)}\right\}$

Numerical scheme and time marching method: The dynamic analysis of structures involves the solution of the well known dynamic equation of motion and sometimes includes the major effects of vertical interfacial joints.

$[M]\{\ddot{\mathrm{u}}\}+[C]\{u\}+[K]\{\dot{u}\}=\left\{f_{(t)}\right\}$

Where $[\mathrm{M}],[\mathrm{C}]$ and $[\mathrm{K}]$ are the mass, damping and stiffness matrices respectively and $\ddot{u}, \dot{u}$ and $\mathrm{u}$ are the displacement, velocity and acceleration of the nods. In direct integration methods the time discretization is directly performed in equation (26), allowing it to obtain the solution for successive time steps. So, in these methods the total earthquake time record is divided into several time steps, $\Delta t$, in which an 
explicit/implicit approximation is applied to the displacements, velocities and accelerations. Basically, the solution progresses knowing the vectors $\ddot{u}_{n}, \dot{u}_{n}$ and $\mathrm{u}^{n}$ at time $\mathrm{t}^{n}$ and calculating the corresponding values at time:

$t_{n+1}=t_{n}+\Delta t$

Dynamic response of boundary value problems: In dynamic analysis of structures, the Newmark direct integration method is frequently used, because it is accurate and unconditionally stable.

Newmark.N.M. in 1959 developed a family of time stepping methods on the basis of the following fundamental relationships:

$\{\dot{u}\}_{t+\Delta t}=\{\dot{u}\}_{t}$

$+\left[(1-\beta)(\ddot{u})_{t}+\beta\{\ddot{u}\}_{t+\Delta t}\right] \Delta t$

$\{u\}_{t+\Delta t}=\{u\}_{t}+\{\dot{u}\}_{t} \Delta t$

$+\left[(0.5-\alpha)\{\ddot{u}\}_{t}+\alpha\{\ddot{u}\}_{t+\Delta t}\right] \Delta t^{2}$

Where the parameters $\alpha$ and $\beta$ are suitably defined in order to obtain a stable and accurate time marching. In fact $\alpha$ and $\beta$ defines the variation of acceleration during the time step. Newmark proposed $\beta=\frac{1}{2}$ and $\frac{1}{6} \leq \alpha \leq \frac{1}{4}$ for unconditional stability. If in the Newmark's equations $\beta=\frac{1}{2}$ and $\alpha=\frac{1}{4}$ are assumed consequently this method is converted to constant acceleration method in which assumes that acceleration values in each time step is not changed. Furthermore if $\beta=\frac{1}{2}$ and $\alpha=\frac{1}{6}$ are assigned then this equals to linear variation of acceleration in each time step.

Solving equation (29) for $\{\ddot{\mathrm{u}}\}_{t+\Delta t}$ and substituting in equation (28) $\{\ddot{\mathrm{u}}\}_{t+\Delta t}$ and $\{\dot{u}\}_{t+\Delta t}$ are obtained as functions of $\{u\}_{t+\Delta t}$ and values at the previous time step. By considering the presence of $\{\ddot{u}\}_{t+\Delta t}$ on the right hand of equation (28) and (29) we have found that this method is an implicit method. Stability condition of Newmark's method is as follows:

$\frac{\Delta t}{T} \leq \frac{1}{\pi \sqrt{2}} \cdot \frac{1}{\sqrt{\beta-2 \alpha}}$

For $\beta=\frac{1}{2}$ and $\alpha=\frac{1}{4}$ the above condition implies $\frac{\Delta t}{T} \leq \infty$ which means that in the case of assuming constant or average acceleration in each time step the Newmark's method is unconditionally stable and just with respect to reception of satisfactory accuracy $\Delta t$ is determined.
Establishing the equilibrium at $\mathrm{t}+\Delta t$, an equation of the form:

$$
\begin{aligned}
& \left.[M]\{\dot{\mathrm{u}}\}_{t+\Delta t}+C\right]_{\{\dot{\mathrm{u}}\}_{t+\Delta t}} \\
& +[K]\{u\}_{t+\Delta t}=\{f(t+\Delta t)\}
\end{aligned}
$$

Can be derived, which can be solved for $\{u\}_{t+\Delta t}$. This procedure is useful in earthquake analysis when accelorograms are used to characterize the ground motion, or when structural nonlinear effects are present. Major steps involved in the solution procedure of the equation (31) based on the Newmark time integration scheme are as follows:

\section{Initial calculations}

i. Form stiffness matrix $[K]$, mass matrix $[M]$ and damping matrix $[C]$.

ii. Initialize $u_{0}, \dot{u}_{0}, \ddot{u}_{0}$.

iii. Select time step $\Delta t$ and parameters $\alpha$ and $\beta$ and evaluate integration constants:

$\mathrm{a}^{0}=\frac{1}{\alpha \Delta t^{2}}$

$\mathrm{a}^{1}=\frac{\beta}{\alpha \Delta t}$

$\mathrm{a}^{2}=\Delta t^{2}\left(\frac{1}{2}-\alpha\right)$

$\mathrm{a}^{3}=\Delta t(1-\beta)$

$\mathrm{a}^{4}=\Delta t . \beta$

iv. Form effective stiffness matrix $\mathrm{K}^{*}$ :

$\mathrm{K}^{*}=\mathrm{K}+\mathrm{a}^{0} \mathrm{M}+\mathrm{a}^{1} \mathrm{C}$

v. Factorizes $\mathrm{K}^{*}$ into Lower, Diagonal and Upper matrices (Transpose of lower):

$\mathrm{K}^{*}=\mathrm{LDL}^{\mathrm{T}}$

\section{For each time step}

i. Calculate predictors (predictor phase):

$d_{n+1}^{[i]}=\tilde{d}_{n+1}=d_{n}+\Delta t \cdot v_{n}+a_{2} \cdot a_{n}$

$v_{n+1}^{[i]}=\tilde{v}_{n+1}=v_{n}+a_{3} \cdot a_{n}$

$a_{n+1}^{[i]}=\left[d_{n+1}^{[i]}-\tilde{d}_{n+1}\right] /\left(\Delta t^{2} \cdot \alpha\right)=0$

Values of , $d_{n+1}^{[i]}, v_{n+1}^{[i]}$ and $a_{n+1}^{[i]}$ are the displacement, velocity and acceleration at time step $\mathrm{n}+1$ and $d_{n}, v_{n}$, $a_{n}$ are the same values at time step $\mathrm{n}$. Also subscript $\mathrm{i}$ indicate the ith iteration at time step $\mathrm{n}$.

ii. Evaluate effective load vectors using equation

$\psi^{[i]}=\psi_{n+1}^{[i]}=f_{n+1}^{[i]}-M a_{n+1}^{[i]}-p\left(d_{n+1}^{[i]}, v_{n+1}^{[i]}\right)$

$\psi_{n+1}^{[i]}, f_{n+1}^{[i]}$ and $p\left(d_{n+1}^{[i]}, v_{n+1}^{[i]}\right)$ are the effective load vector, external load vector and internal load vector at 
time step $n+1$ respectively. In the equation (36) the internal force values are

$$
p=\int_{v} B^{T} \sigma d v
$$

iii) Solve for incremental displacement at time step $n+1$ by using:

$\mathrm{K}^{*} \Delta d^{[i]}=\psi^{[i]}$

iv. Calculate displacements, velocities and accelerations at time step $\mathrm{n}+1$ (corrector phase):

$d_{n+1}^{[i+1]}=\tilde{d}_{n+1}+\Delta d^{[i]}$

$a_{n+1}^{[i+1]}=\left[d_{n+1}^{[i+1]}-\tilde{d}_{n+1}\right] \cdot a_{0}$

$v_{n+1}^{[i+1]}=\tilde{v}_{n+1}+a_{4} \cdot a_{n+1}^{[i+1]}$

v. If $\Delta d^{[i]}$ and/or $\psi^{[i]}$ do not satisfy the convergence conditions then set $\mathrm{i}=\mathrm{i}+1$ and go to step (ii), otherwise continue.

vi. Set

$$
\begin{aligned}
& d_{n+1}=d_{n+1}^{[i+1]} \\
& v_{n+1}=v_{n+1}^{[i+1]} \\
& a_{n+1}=a_{n+1}^{[i+1]}
\end{aligned}
$$

vii. Set $n=n+1$ and repeat steps (i) to (vii).

Element library: The several 3D solid finite $(8,12,16$, 20 nodded ), infinite ( $8,12,16$ nodded ) and joint elements $(8,12,16$ nodded) can be available in the program are the well known isoparametric types of elements. Each elements or combination of different elements are used in the finite element model with a pre-assigned code number specified by the programmer. In each node of the mentioned elements three translation movements in $\mathrm{x}, \mathrm{y}$ and $\mathrm{z}$ direction of Cartesian space are considered. Furthermore it is worth to say that the ability of combination of $1 \mathrm{D}$ and $3 \mathrm{D}$ elements is prepared into the software.

Material Library: The material models available in the program are listed as follows:

\section{Elastic}

a. Solid: Elastic - Perfectly Plastic

Elastic - Plastic with Linear Strain hardening

Elastic

b. Joint: Elastic - Perfectly Plastic

Elastic - Plastic with Linear Strain hardening

Software development: The software is of multi element features, this is achieved by identifying each element, by a particular code number. Based upon this assigned element code number, number of nodes, per element, order of integration, shape functions and their derivations were picked up. The Jacobin $[J]$ matrix, its inverse $[J]^{-1}$, Elasticity $[D]$ matrix., Strain displacement $[\mathrm{B}]$ matrix, Stiffness $[K]$ matrix, Mass $[M]$ matrix and Damping $[C]$ matrix were therefore, computed automatically. Furthermore this software can be used for both static and dynamic loads. This may be achieved by entering a code number into the software that assigned kind of interested analysis: $\mathrm{NOA}=1$ (static analysis) and $\mathrm{NOA}=2$ (dynamic analysis).

The procedures followed by the package for each analysis are summarized in Fig. 2 and 3.

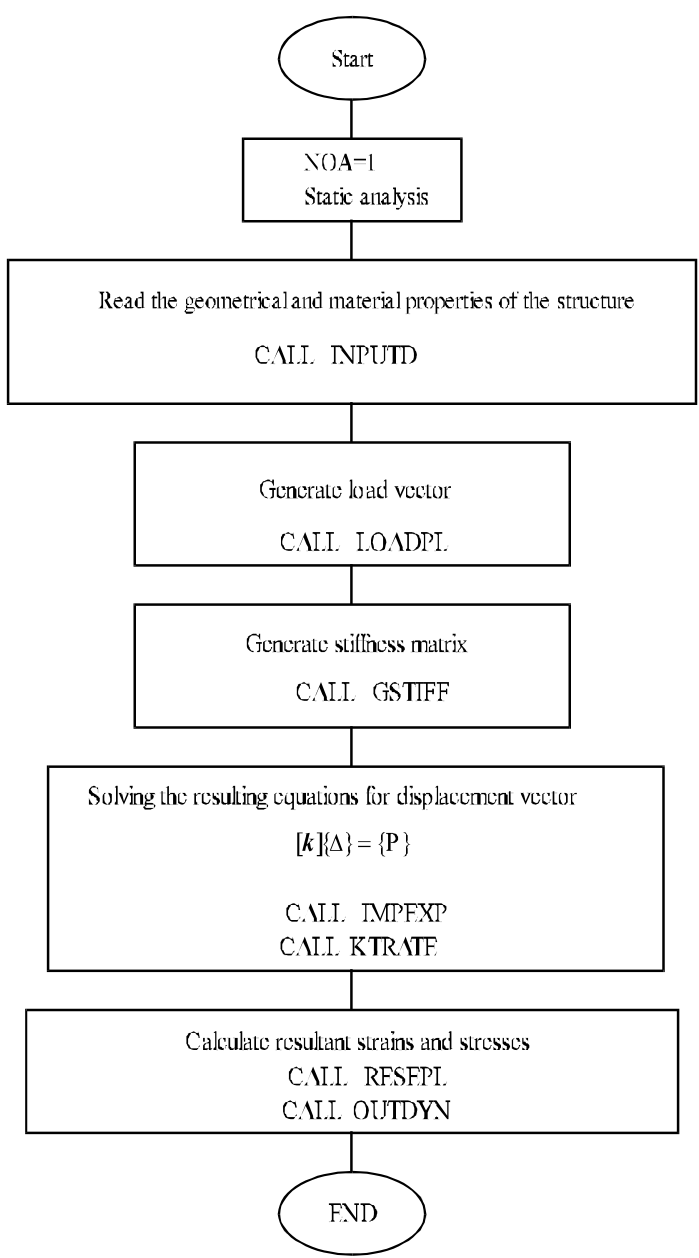

Fig. 2: Flowchart of static procedure of the proposed software

Validation tests of the software: Some of the bench mark tests which have been conducted by a number of authors or their close form solution are reanalyzed for both static and dynamic types of analysis in order to demonstrate the validation of the package developed.

\section{A. Static phase}

Simply supported beam subjected to a static concentrated loads: Figure 4 Show a simply supported beam subjected to concentrated load at its mid span. 


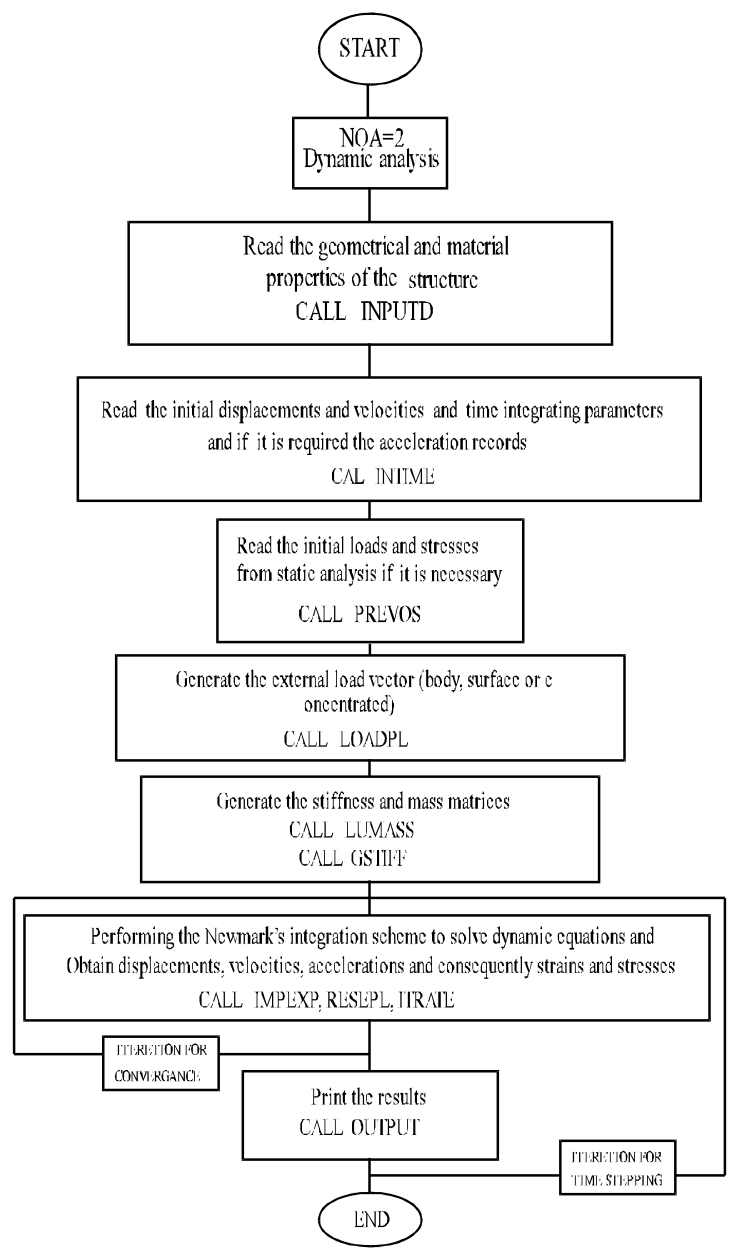

Fig. 3: Flowchart of dynamic procedure of the proposed software

The Finite Element discretisation along with material and geometrical properties are also presented in Fig. 4.

The maximum displacement and bending stress evaluated from the present study and classical strength of material are shown in Table 3. From the Comparison of results has shown that there is a good agreement between the theoretical and calculated results from the present study.

Table 3: Comparison of maximum displacement and bending stress

\begin{tabular}{lll}
\hline Item & Theory & Present Study \\
\hline Maximum bending Stress (Mpa) & 16.67 & 16.89 \\
Maximum displacement (cm) & 0.185 & 0.184 \\
\hline
\end{tabular}

Cantilever beam subjected to static axial loads: Figure 5 shows the Finite Element discretization of a Cantilever beam subjected to an axial load at the free end of the beam.

Furthermore, to study the joint element behavior under loads acting perpendicular to its plane a joint element has been inserted in the middle part of the beam and then the problem is analyzed for two different normal stiffness values namely:
Case I: No interfacial element

$$
\mathrm{K}_{\mathrm{nn}}=2 \times 10^{5}\left(\mathrm{~kg} / \mathrm{cm}^{2}\right) \quad \mathrm{K}_{\mathrm{ss}}=2 \times 10^{5}\left(\mathrm{~kg} / \mathrm{cm}^{2}\right)
$$

Case II: Interfacial element

$$
\mathrm{K}_{\mathrm{nn}}=10\left(\mathrm{~kg} / \mathrm{cm}^{2}\right)
$$

Case III: Interfacial element

$$
\mathrm{K}_{\mathrm{nn}}=5\left(\mathrm{~kg} / \mathrm{cm}^{2}\right) \quad \mathrm{K}_{\mathrm{ss}}=2 \times 10^{5}\left(\mathrm{~kg} / \mathrm{cm}^{2}\right)
$$

The plot of axial displacements along the length of the beam is illustrated in Fig. 6. It can be seen from this illustration that for the case I in which the high values of $\mathrm{K}_{\mathrm{n}}$ are used there is a continuous curve and axial displacements vary smoothly along the length of the beam. But when low values of $\mathrm{K}_{\mathrm{nn}}$ is assigned as the case II and III then there is a drop in axial displacement in the location of the interfacial element. Therefore we can see that proposed model has the ability of good prediction of physical behavior of joint materials inserted to the beam. Furthermore to show the agreement between theoretical and obtained results from model the analytical axial displacement of the free end of the beam has been brought here:

For the case III which $k_{n n}=5\left(\frac{\mathrm{kg}}{\mathrm{cm}^{2}}\right)$ we have:

Total axial displacement at the free end of the beam with respect to Fig. 7 is:

$\delta_{a}=\delta_{a 1}+\delta_{a 2}+\delta_{a 3}$

$\delta_{a 1}=\frac{P L}{A E}=\frac{100000 \times 100}{(20 \times 30) \times 2 \times 10^{5}}=0.0833 \mathrm{~cm}$

$\delta_{a 2}=\frac{P}{K}=\frac{100000}{5 \times(20 \times 30)}=33.333 \mathrm{~cm}$

$\delta_{a 3}=\frac{P L}{A E}=\frac{100000 \times 100}{(20 \times 30) \times 2 \times 10^{5}}=0.0833 \mathrm{~cm}$

$\delta_{a}=0.0833+33.333+0.0833=33.50 \mathrm{~cm}=0.335 \mathrm{~m}$

With comparing this result to that obtained from model in the Fig. 6 it can be seen that there is good agreement between them.

Cantilever beam subjected to static vertical load at its free end: For study the behavior of proposed successive joint element under loads acting tangential to its plane this verification is considered.

The plot of vertical displacements along the length of the beam is illustrated in Fig. 9. It can be seen from this illustration that for the case I in which the high values of $\mathrm{K}_{\mathrm{ss}}$ are used there is a continuous curve and vertical displacements vary smoothly along the length of the beam. But when low values of $\mathrm{K}_{\mathrm{ss}}$ is assigned as the case II and III then there is a drop in vertical displacement in the location of the interfacial element. Therefore we can see that proposed model has the ability of good prediction of physical behavior of joint materials inserted to the beam.

Furthermore to show the agreement between theoretical and obtained results from model the 

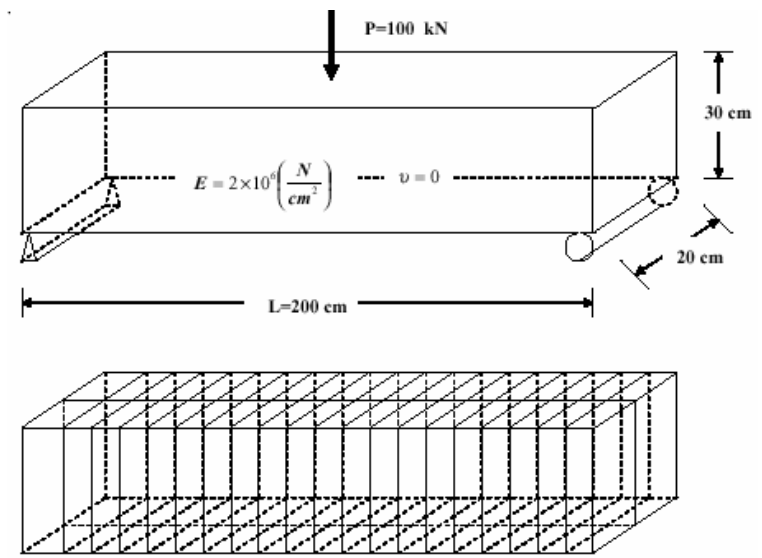

Fig. 4: Simply supported beam subjected to static concentrated load

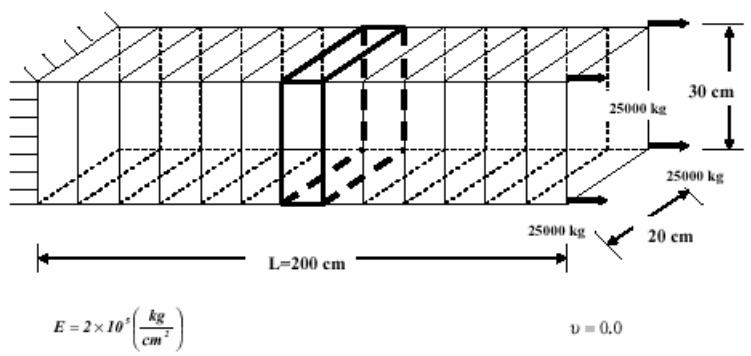

Fig. 5: Cantilever beam subjected to a static concentrated load

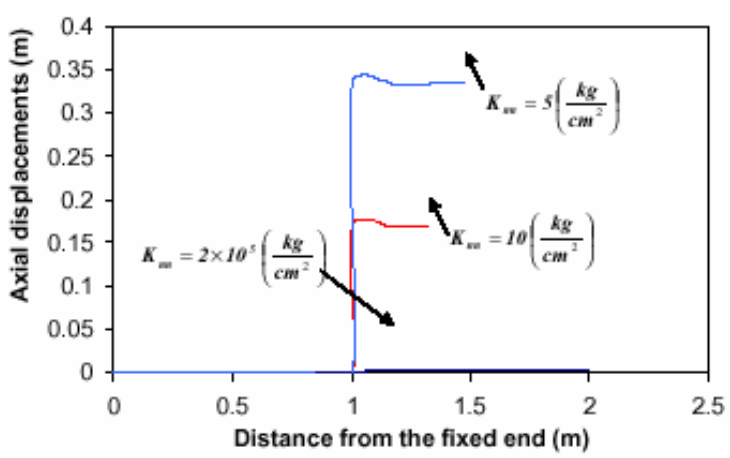

Fig. 6: Plot of axial displacements along the length of the cantilever beam

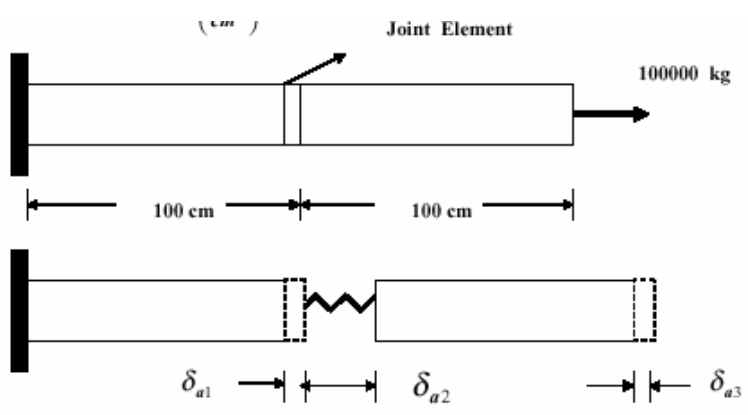

Fig. 7: Analytical model of the beam

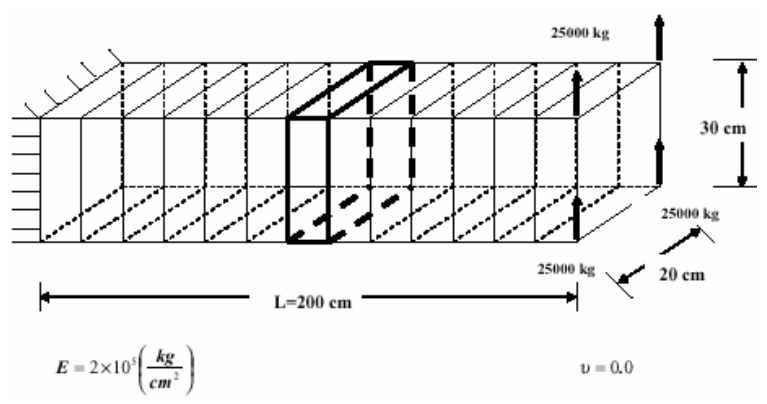

Fig. 8: Cantilever beam subjected to a static concentrated load

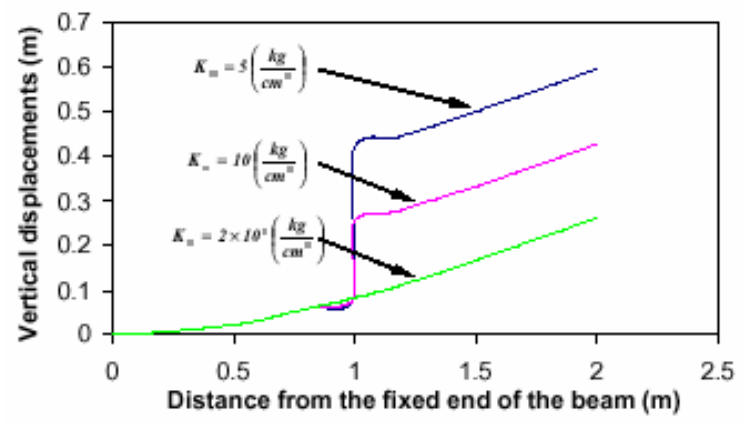

Fig. 9: Plot of vertical displacements along the length of the cantilever beam

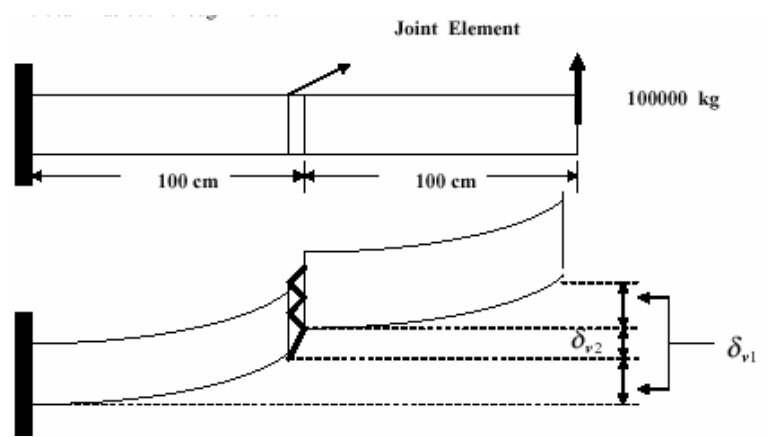

Fig. 10: Analytical model of the beam

analytical axial displacement of the free end of the beam has been brought here:

For the case III which $k_{s s}=5\left(\frac{\mathrm{kg}}{\mathrm{cm}^{2}}\right)$ we have:

Total vertical displacement at the free end of the beam with respect to Fig. 10 is:

$$
\begin{aligned}
& \delta_{v}=\delta_{v 1}+\delta_{v 2}, \delta_{v 1}=\frac{P L^{3}}{3 E I}=\frac{100000 \times 200^{3} \times 12}{3 \times 2 \times 10^{5} \times 20 \times 30^{3}}=29.63 \mathrm{~cm} \\
& \delta_{v 2}=\frac{P}{K}=\frac{100000}{5 \times(20 \times 30)}=33.333 \mathrm{~cm} \\
& \delta_{v}=29.63+33.333=62.96 \mathrm{~cm}=0.629 \mathrm{~m}
\end{aligned}
$$

With comparing this result to that obtained from model in the Fig. 9 it can be seen that there is good agreement between them. 


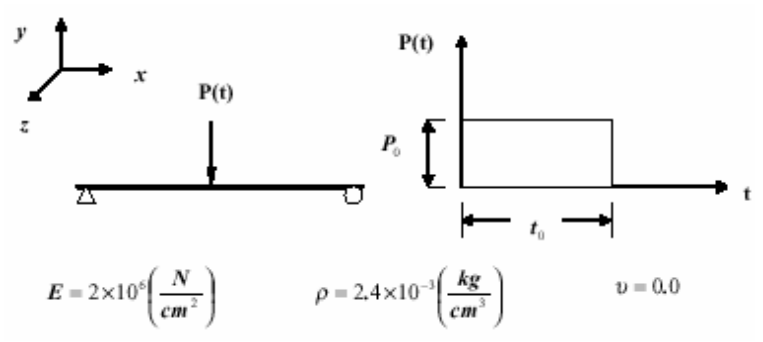

Fig. 11: Simply supported beam under impact concentrated load

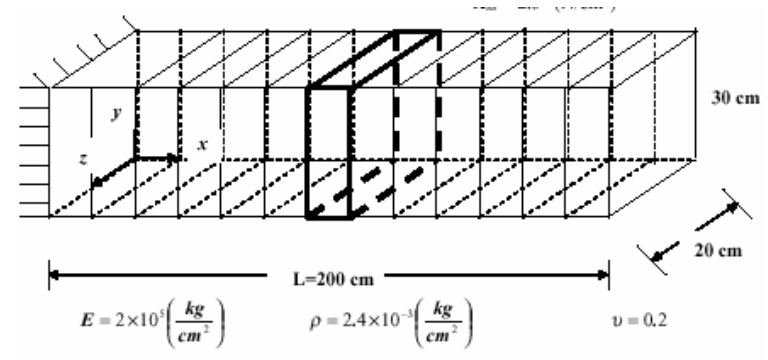

Fig. 12: Cantilever beam subjected to support excitation

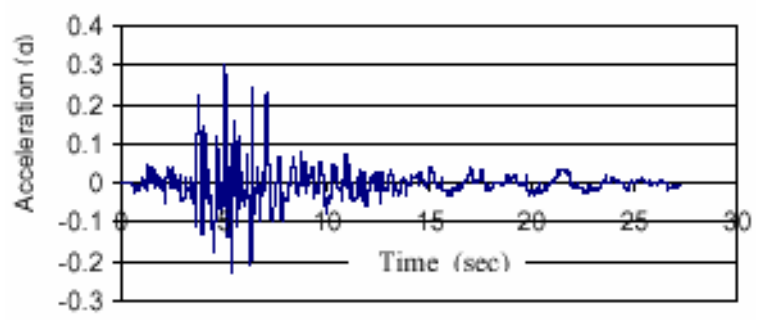

Fig. 13: Earthquake record in z-direction

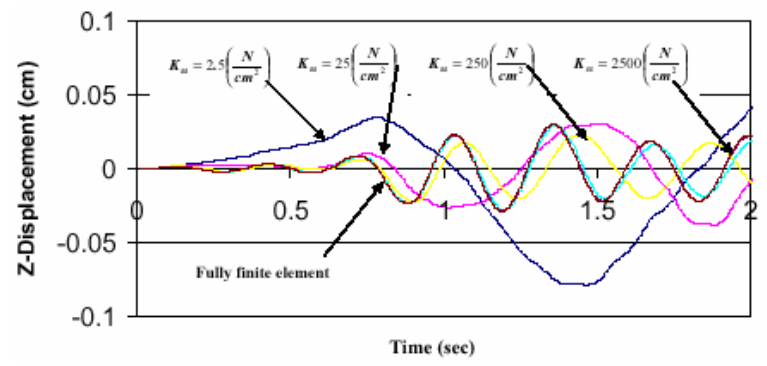

Fig. 14: Displacement time history of the free end of the cantilever beam

\section{B. Dynamic phase}

Simply supported beam under single dynamic point loads: A simply supported beam subjected to a concentrated suddenly point load applied at the middle of its span has been analyzed (Fig. 11).

Finite element modeling and geometric properties of this beam as the same we have seen in bench mark test 9.1 .

Table 4: Good agreement between the results obtained Item Close Form Solution Present Study $\begin{array}{lll}\text { Maximum displacement } & 0.400 & 0.403\end{array}$

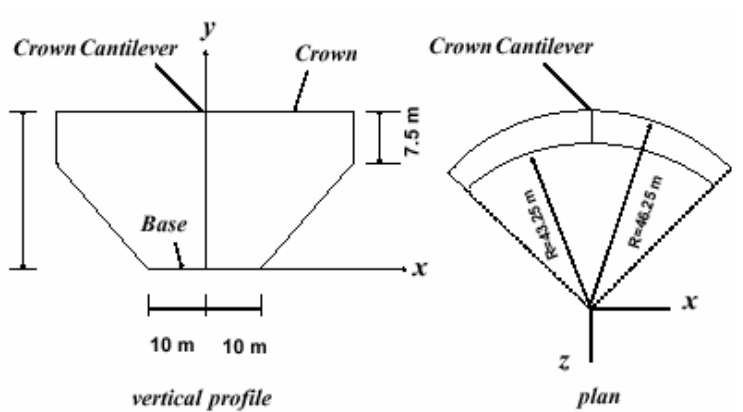

Fig. 15: Geometry dimensional of the arch dam type-I U.S.B.R

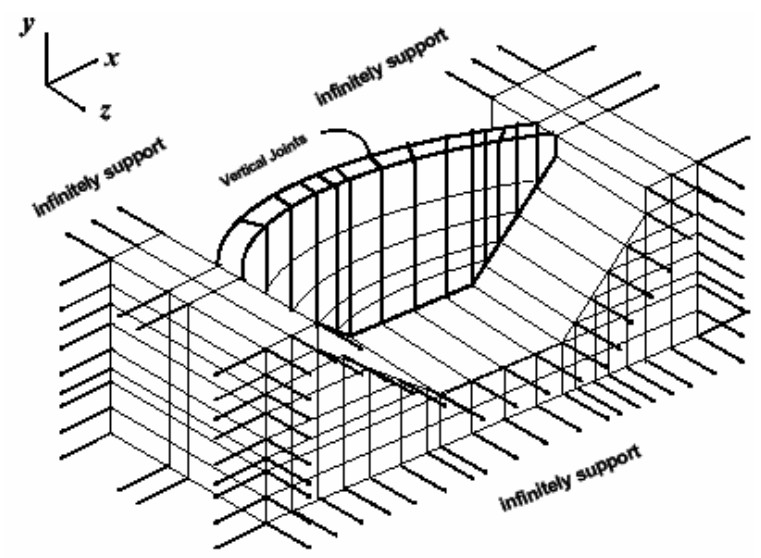

Fig. 16: Finite element idealization of the arch dam type-I U.S.B.R

Cantilever beam subjected to earthquake excitation: In order to verify the validity of the interface element in time domain, a cantilever beam subjected to earthquake record in $\mathrm{z}$ - direction at its support is analyzed.

The geometrical, material properties and the finite element discretization of the beam are shown in Fig. 12.

Also the earthquake record that used for analysis is shown in Fig. 13.

The displacement time history of the free end nodal point is plotted in Fig. 14 for the following cases:

I. Fully finite element

II. $\mathrm{K}_{\mathrm{nn}}=2.5 \mathrm{E} 6\left(\mathrm{~N} / \mathrm{cm}^{2}\right) \mathrm{K}_{\mathrm{ss}}=2500\left(\mathrm{~N} / \mathrm{cm}^{2}\right)$

III. $\mathrm{K}_{\mathrm{nn}}=2.5 \mathrm{E} 6\left(\mathrm{~N} / \mathrm{cm}^{2}\right) \mathrm{K}_{\mathrm{ss}}=250\left(\mathrm{~N} / \mathrm{cm}^{2}\right)$

IV. $\mathrm{K}_{\mathrm{nn}}=2.5 \mathrm{E} 6\left(\mathrm{~N} / \mathrm{cm}^{2}\right) \mathrm{K}_{\mathrm{ss}}=25\left(\mathrm{~N} / \mathrm{cm}^{2}\right)$

V. $\mathrm{K}_{\mathrm{nn}}=2.5 \mathrm{E} 6\left(\mathrm{~N} / \mathrm{cm}^{2}\right) \mathrm{K}_{\mathrm{ss}}=2.5\left(\mathrm{~N} / \mathrm{cm}^{2}\right)$

It is clear form this figure that, there is almost identical response of the beam for the case of (i) to (iv), while there is a deviation in response of the beam when case to (iv) are considered i.e. amplitude of vibration increases with reduction in value of $\mathrm{k}_{\mathrm{ss}}$.

Application of proposed model to the practical problem: The numerical example that was elected here is an arch dam type-I, according to U.S.B.R classification with constant thickness and radius shown in Fig. 15.

Material properties of the dam and its supporting system are as follows: 


$$
\begin{array}{cc}
E_{c}=3 \times 10^{4}(\mathrm{MPa}) & v_{c}=0.2 \\
f_{c}^{\prime}=40(\mathrm{MPa}) & \rho_{c}=2400\left(\frac{\mathrm{kg}}{\mathrm{m}^{3}}\right) \\
E_{R}=3 \times 10^{4}(\mathrm{MPa}) & v_{R}=0.2 \\
f_{R}^{\prime}=40(\mathrm{MPa}) & \rho_{R}=2400\left(\frac{\mathrm{kg}}{\mathrm{m}^{3}}\right)
\end{array}
$$

Crack resistance of concrete as $0.44\left(f_{c}^{\prime}\right)^{\frac{2}{3}}$ is considered. Normal and shear stiffness of contraction joints are $K_{n n}=50 E_{c}$ and $K_{s s}=0.2 E_{c}$.

In dynamic analysis $f_{c}^{\prime}$ is increased up to $30 \%$.

The finite element idealization of the problem under static and dynamic loading is depicted in Fig. 16.

Here first the static analysis for the dead weight has been performed, the stress and load vector are stored for seismic response. The seismic response of the dam has been carried out for the upstream-downstream component of Zangiran earthquake $19^{\text {th }}$ of June 1994 that was shown before in Fig. 13.

\section{CONCLUSION}

The software which is presented in this study is multi-element software with several types of solid, 3-D finite, infinite and interface element in its elements library. The program can be employed in a small personal computer to analyze a large problem. It equipped with special memory allocation and management.

It is also dual purposes which can simultaneously analyze structure under static and dynamic loads.

\section{REFERENCES}

1. Zienkiewicz, O.C., 1983. Finite Element Method. McGraw-Hill.

2. Noorzaei, J., 1995. Concepts and application of three dimensional infinite elements to soil structure interaction problems. Intl. J. Engg., 9: 131-142. 